\title{
Quantum Spin Lenses in Atomic Arrays
}

\author{
A. W. Glaetzle, ${ }^{1,2,3,4}$ K. Ender, ${ }^{1,2}$ D. S. Wild, ${ }^{5}$ S. Choi, ${ }^{5}$ H. Pichler, ${ }^{6,5}$ M. D. Lukin, ${ }^{5}$ and P. Zoller ${ }^{1,2,7}$ \\ ${ }^{1}$ Institute for Theoretical Physics, University of Innsbruck, A-6020 Innsbruck, Austria \\ ${ }^{2}$ Institute for Quantum Optics and Quantum Information of the Austrian Academy of Sciences, \\ A-6020 Innsbruck, Austria \\ ${ }^{3}$ Centre for Quantum Technologies, National University of Singapore, \\ 3 Science Drive 2, Singapore 117543 \\ ${ }^{4}$ Clarendon Laboratory, University of Oxford, Parks Road, Oxford OX1 3PU, United Kingdom \\ ${ }^{5}$ Department of Physics, Harvard University, Cambridge, Massachusetts 02138, USA \\ ${ }^{6}$ ITAMP, Harvard-Smithsonian Center for Astrophysics, Cambridge, Massachusetts 02138, USA \\ ${ }^{7}$ Max-Planck-Institut für Quantenoptik, 85748 Garching, Germany
}

(Received 28 April 2017; revised manuscript received 9 July 2017; published 20 September 2017)

\begin{abstract}
We propose and discuss quantum spin lenses, where quantum states of delocalized spin excitations in an atomic medium are focused in space in a coherent quantum process down to (essentially) single atoms. These can be employed to create controlled interactions in a quantum light-matter interface, where photonic qubits stored in an atomic ensemble are mapped to a quantum register represented by single atoms. We propose Hamiltonians for quantum spin lenses as inhomogeneous spin models on lattices, which can be realized with Rydberg atoms in 1D, 2D, and 3D, and with strings of trapped ions. We discuss both linear and nonlinear quantum spin lenses: in a nonlinear lens, repulsive spin-spin interactions lead to focusing dynamics conditional to the number of spin excitations. This allows the mapping of quantum superpositions of delocalized spin excitations to superpositions of spatial spin patterns, which can be addressed by light fields and manipulated. Finally, we propose multifocal quantum spin lenses as a way to generate and distribute entanglement between distant atoms in an atomic lattice array.
\end{abstract}

DOI: 10.1103/PhysRevX.7.031049

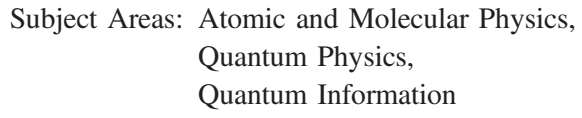

Subject Areas: Atomic and Molecular Physics, Quantum Physics, Quantum Information

\section{INTRODUCTION}

In quantum information processing [1] with atoms, qubits are typically represented by internal atomic states, e.g., as long-lived spin excitations within the atomic ground-state manifold [2]. Ideally, qubits are stored in single atoms, and for these qubits to be identifiable and addressable, we typically require localization of the atoms in well-defined spatial regions. Spatial control and localization of single atoms is a prerequisite to implement single- and two-qubit operations, allowing addressing of individual qubits with laser light, and providing entangling operations between adjacent qubits by finite-range interactions. Recent atomic physics experiments have demonstrated in a remarkable way the basic ingredients of single atom manipulation and addressing with trapped atoms and ions, and controlled interaction and entanglement between atomic spin qubits with Rydberg atoms [3,4], trapped ions [5-8], cavity QED setups [9], and quantum interfaces [10-12].

Published by the American Physical Society under the terms of the Creative Commons Attribution 4.0 International license. Further distribution of this work must maintain attribution to the author(s) and the published article's title, journal citation, and DOI.
In contrast to localized qubits stored in single trapped atoms, atomic ensembles provide us with qubits in the form of delocalized spin excitations [13,14]. Delocalized spin qubits arise naturally in light-atomic ensemble interfaces in both free space and cavity-assisted setups. Here, incident photons representing a "flying qubit" are absorbed in an atomic ensemble with enhanced interactions benefiting from a large atom number $N$, as in an optically thick medium, and converted into a spin excitation, which may be delocalized over the whole atomic cloud [15-19]. In order to create controlled interactions between such delocalized qubits it is desirable to convert delocalized spin qubits into localized qubits in the atomic array representing quantum memory. Thus, ideally we want operations-a lens for spin excitations - on the atomic array, which allow in a coherent process "focusing" of qubits to a well-defined and localized region, and ultimately to a single atom.

In this paper, we propose and discuss linear and nonlinear quantum spin lenses and their physical realization in quantum optical setups. We first identify Hamiltonians to realize linear spin lenses, which map in a coherent process a delocalized to localized spin excitation, and vice versa. This has immediate application as a quantum atom-light interface, where incident photonic qubits are sequentially stored in an atomic array and focused to a quantum register of 

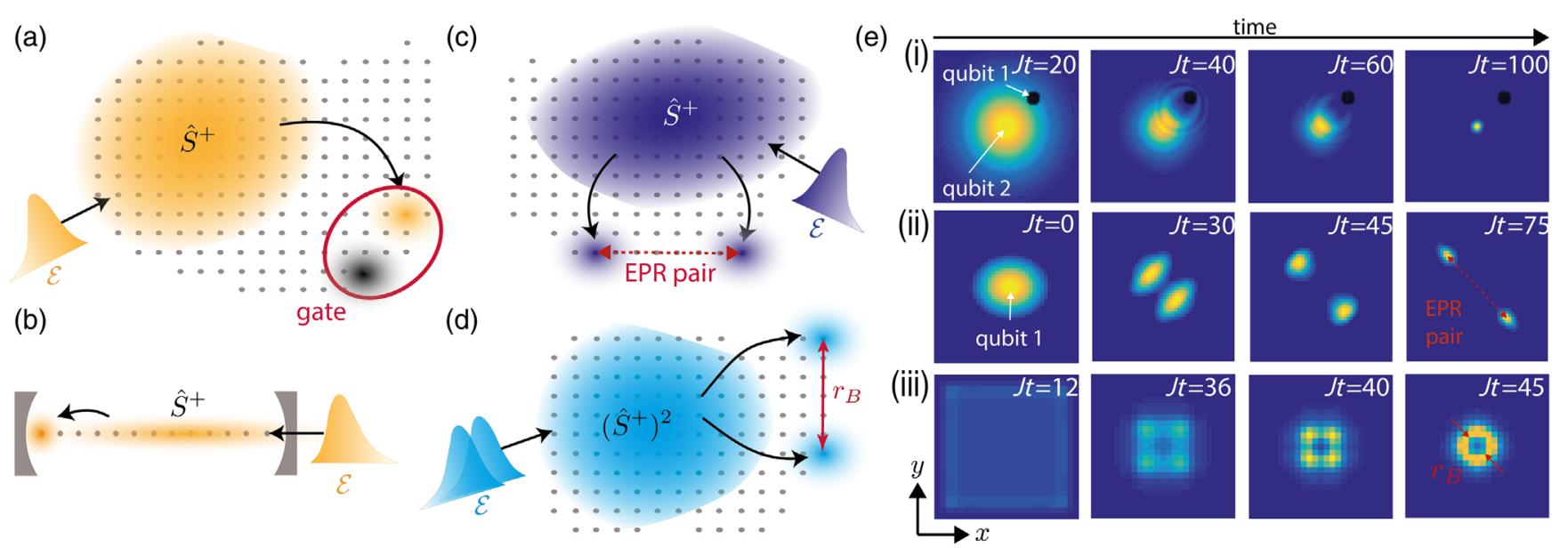

FIG. 1. (a)-(d) Various scenarios of a quantum light-atom interface illustrating a quantum spin lens. Incident photonic qubits are initially stored as delocalized spin excitations in an atomic array, and focused to single atoms (see Sec. II). (a) Basic process of write and focusing operations (see Secs. II A and II B). Qubits stored in single atoms allow quantum gate operations to be implemented between adjacent atoms, e.g., via Rydberg gates. (b) 1D setup with atomic ensemble stored inside a cavity (see Sec. II D). (c) Generation of EPRtype states by constructing lenses with multiple focal points (see Sec. II C). (d) Focusing dynamics with a nonlinear spin lens with repulsive spin-spin interactions with range $r_{B}$, illustrated for two spin excitations (see Sec. III). (e) Density plots illustrating focusing dynamics of spin excitations in 2D arrays as a function of time $t$ according to spin-lens Hamiltonians Eqs. (1) or (10). (i) Focusing of an initially delocalized "qubit 2" in the presence of a hole (representing, e.g., a previously stored "qubit 1") (see Sec. II). (ii) Two-focus lens illustrating generation of EPR pairs (see Sec. IIC). Parameters: $50 \times 50$ lattice, initial width of the Gaussian wave packet $\sigma_{0}=10 a$ lattice spacings. (iii) Focusing of two spin excitations with a nonlinear quantum lens with repulsive spin-spin interactions of range $r_{B}$. The two excitations are focused to a ring, reminiscent of a quantum crystal. Parameters: $31 \times 31$ lattice $($ see Sec. III).

spatially localized spin qubits represented by single atoms [cf. Fig. 1(a)] with coherent transport dynamics [20]. Moreover, we can generalize the concept of the quantum spin lens to a multifocal lens. In particular, this allows a single delocalized spin excitation to be mapped to a spatial EPR-like superposition state, thus providing a way to distribute or generate entanglement between (distant) atoms [cf. Fig. 1(c)]. Finally, we discuss the design of nonlinear spin lenses, adding finite-range (repulsive) spin-spin interactions to the spin-lens Hamiltonian. Thus, focusing dynamics is conditional on the number of initial spin excitations, and an initial quantum superposition state of delocalized spins is mapped to a superposition of spatial spin patterns [cf. Fig. 1(d)]. Remarkably, this provides a tool to manipulate the individual terms (corresponding to a specific excitation number) in the superposition state by spatial addressing in the atomic medium. As noted above, the relevant spin models are naturally implemented in existing atomic and solid-state quantum optical setups, and we illustrate this below with the examples of neutral atoms with Rydberg-mediated spin-spin interactions in 1D, 2D, and 3D atomic lattices [21-24] using laser-dressing techniques [25-28], as well as with strings of trapped ions [7,8].

\section{LINEAR QUANTUM SPIN LENSES: FOCUSING DYNAMICS OF SINGLE SPIN EXCITATIONS}

We are interested here in a scenario illustrated in Fig. 1(a), where an incident wave packet $\mathcal{E}$, representing a qubit $\alpha|0\rangle+\beta|1\rangle$ as a superposition of vacuum and a one-photon wave packet, is stored as a delocalized spin excitation in a medium of $N$ two-level atoms. These two-level systems can be physically represented by long-lived atomic hyperfine ground states two-level atoms $|g\rangle,|e\rangle$, with all atoms initially prepared in the ground state, and we assume atoms trapped in an array. Storage of a photonic qubit in the atomic medium is achieved, for example, in a Raman process $[29,30]$, where the incident photon is absorbed and atoms initially prepared in $|g\rangle$ transfer to $|e\rangle$. Writing to atomic quantum memory thus corresponds to a mapping of the photonic qubit to the atomic state $\alpha|G\rangle+\beta \hat{S}^{+}|G\rangle$. Here, $\hat{S}^{+}=\sum_{n=1}^{N} \psi_{n} \hat{\sigma}_{+}^{(n)}$, a sum of Pauli-raising operators for atoms $n$, creates a delocalized excitation distributed over the atoms according to an amplitude $\psi_{n}$, acting on the vacuum state $|G\rangle \equiv\left|g_{1}, \ldots, g_{N}\right\rangle$ with all atoms in the ground state. For a low enough excitation density in the atomic medium these spin excitations are essentially noninteracting and behave as bosons up to corrections $\sim 1 / N$.

A quantum spin lens aims to achieve a mapping of the delocalized atomic spin excitation to (ideally) a single atom, $\hat{S}^{+}|G\rangle \rightarrow \hat{\sigma}_{+}^{\left(n_{f}\right)}|G\rangle$ in a coherent quantum process, and preserving the superposition character of the qubit. Below, we first discuss spin-lens Hamiltonians that focus a single initially delocalized spin excitation during the associated unitary time evolution, which we call a linear spin lens. Nonlinear spin lenses which focus multiple interacting spin excitations are discussed in the following section. 
The focusing of single spin excitations discussed below can be generalized immediately to $k$ photonic qubits, provided we store and focus them sequentially, i.e., incident photonic qubits are absorbed and focused in the atomic medium one by one in spatially separated atoms $n_{1}, \ldots, n_{\nu}$ representing a quantum register [31]. Because of the spatial localization, these atomic qubits can now be individually addressed, and we can operate on them with single- and two-qubit gate operations, implemented, for example, as Rydberg gates [see Fig. 1(a) and panel (i) of Fig. 1(e)].

\section{A. Spin-lens Hamiltonian with nearest-neighbor flip-flop interactions}

Focusing of a delocalized excitation in a spin chain is achieved with the Hamiltonian

$$
\hat{H}=-J \sum_{n}\left[\hat{\sigma}_{+}^{(n)} \hat{\sigma}_{-}^{(n+1)}+\text { H.c. }\right]+\sum_{n} V_{n} \hat{\sigma}_{z}^{(n)},
$$

where $\hat{\sigma}_{\mu}^{(n)}$ are Pauli spin-1/2 operators at lattice site $n$. The first term describes hopping of the spin excitation (kinetic energy), which for the moment we take as nearest-neighbor hopping, while the second term is a spatially dependent energy shift $V_{n}=v_{0}\left(n-n_{f}\right)^{2}$. While in Eq. (1) we write a 1D model, the present discussion can in a straightforward way be generalized to higher dimensions.

The Hamiltonian Eq. (1) is motivated by analogy to an optical lens with $V_{n}$ imprinting a phase on the $n$th spin centered around lattice site $n_{f}$, reminiscent of the refractive material of a lens $[32,33]$. The analogy to an optical lens is best illustrated by visualizing the focusing dynamics of single spin excitations with a Wigner phase space distribution as a function of time [see Fig. 1(e) and upper panels of Figs. 2(a) and 2(b)]. We write the wave function of the single spin excitation as $|\psi(t)\rangle=\sum_{n} \psi_{n}(t) \hat{\sigma}_{+}^{(n)}|G\rangle$ with amplitude $\psi_{n}(t)$ initially delocalized as a wave packet of spatial width $\sigma_{0}$ over the lattice, and we define a Wigner function on the lattice as $[34,35](\hbar=1)$

$$
W_{\text {lat }}\left(x_{n}, k\right)=\frac{a}{\pi} \int_{-\pi / 2 a}^{\pi / 2 a} d q\langle k-q \mid \psi\rangle\langle\psi \mid k+q\rangle e^{-2 i q x_{n}} .
$$

Here, $x_{n}=$ an $(n \in \mathbb{Z})$ are discrete lattice positions with $a$ the lattice spacing, and momentum $k a \in(-\pi, \pi)$ is $2 \pi$ periodic, and we denote by $|k\rangle=(a / 2 \pi)^{1 / 2} \sum_{n} e^{i k x_{n}} \hat{\sigma}_{+}^{(n)}|G\rangle$ spin waves with momentum $k$ on an infinite lattice. A momentum space representation of the time-dependent Schrödinger equation with Hamiltonian Eq. (1) shows that the dynamics is the one of a quantum pendulum. The first term in Eq. (1) gives rise to a Bloch band dispersion relation $\epsilon(k)=2 J[1-\cos (k a)]$, and the quadratic potential term maps to a Laplacian in $k$, i.e., a kinetic energy term.

Focusing dynamics is best illustrated in the continuum limit, i.e., we assume that the spin dynamics is smooth on the scale of the lattice spacing, and the wave function in momentum space remains localized to a region at the bottom of the Bloch band. Thus, the dispersion relation is well approximated by $\epsilon(k) \approx J(k a)^{2}+\mathcal{O}(k a)^{4}$ for small momenta $k a \ll 1$, and the Wigner function $W_{\text {lat }}\left(x_{n}, k\right)$ maps to the standard Wigner function $W(x, p)$ for the continuous variables $x_{n} \rightarrow x \in \mathbb{R}$ and $k \rightarrow p \in \mathbb{R}$. The Hamiltonian Eq. (1) becomes an effective harmonic oscillator, $H=p^{2} /(2 m)+m \omega^{2} x^{2} / 2$, with momentum $p$ and position $x$. Here, we define a frequency $\omega=$ $2 \sqrt{v_{0} J}$, mass $m=1 /\left(2 J a^{2}\right)$, and we denote by $\ell=$ $(\hbar / m \omega)^{1 / 2}$ the harmonic oscillator length. Under this Hamiltonian an initial Wigner function, $W(x, p ; 0)$, simply performs a (classical) rigid rotation in phase space, $W(x, p ; t)=W(\bar{x}(x, p, t), \bar{p}(x, p, t) ; 0)$, where position, $\bar{x}(x, p, t)=m \omega x \cos \omega t-p \sin \omega t$, and momentum, $\bar{p}(x, p, t)=p \cos \omega t+m \omega x \sin \omega t$, describe elliptical trajectories in phase space as a function of time. Thus, an initial
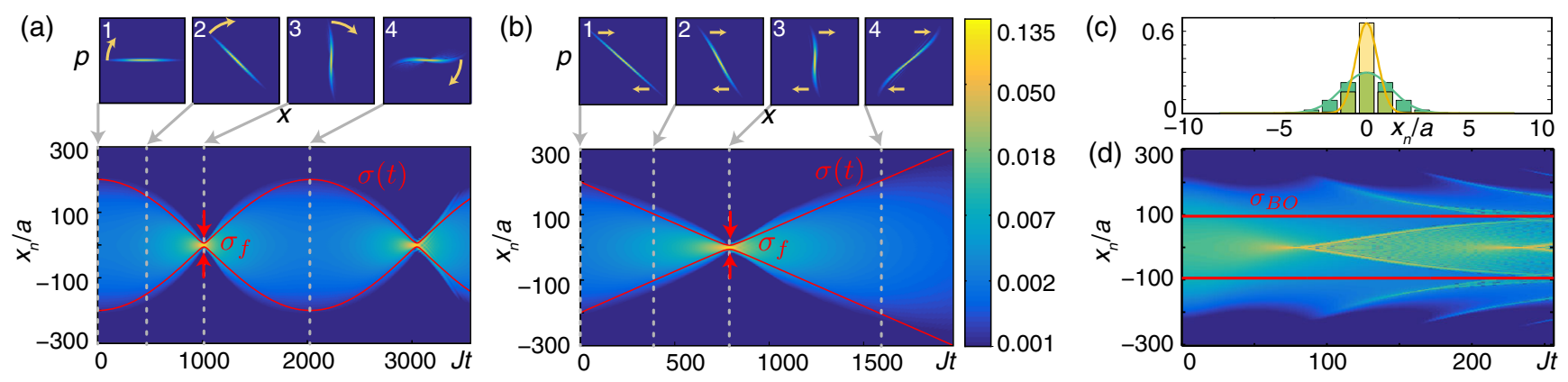

FIG. 2. Time evolution of an initially delocalized spin excitation on a lattice according to the spin-lens Hamiltonian Eq. (1) for the (a) thick and (b) thin lens for a 1D string of $L=800$ spins as a function of time $t$. The red line, $\sigma(t)$, indicates the continuum result. Insets 1-4 above illustrate the dynamics of the Wigner function at four specific times. (c) Zoom to illustrate the final optimized spatial excitation probability per atom illustrating that focusing on the scale of (essentially) single atoms can be obtained. The green bars correspond to a focusing dynamics of the thick lens with initial width $\sigma_{0}=100 a$ and final width $\sigma_{f}=2.7 a$. The yellow bars correspond to a second focusing stage with a thin lens starting with $\sigma_{0}=2.7 a$ to final width $\sigma_{f}=1.2 a$. (d) Bloch oscillations in focusing dynamics at the edge $\sigma_{\mathrm{BO}}$ of the lens (see text). 
wave packet with width $\sigma_{0}$ in position space is transformed after a quarter of period, $t_{f}=\pi /(2 \omega)$, to a spatially localized state with width $\sigma_{f}=\ell^{2} / \sigma_{0} \ll \ell$, as familiar from squeezed states [2,36].

In this continuum approximation the single particle Schrödinger equation from Eq. (1) is formally equivalent to the paraxial Helmholtz equation [33]. The role of time in the Schrödinger equation is replaced by the axial dimension in the paraxial Helmholtz equation, and the potential translates to a spatially dependent refractive index. This allows us to interpret most of the focusing dynamics in the language of classical optics. So far, we consider focusing of a delocalized excitation in a potential $V_{n}$, which is "on" during the whole dynamics. In analogy to optics this corresponds to light propagating in a graded index multimode fiber. In the following, we refer to this dynamics as a thick lens. This is to distinguish from a second scenario, discussed below, where focusing is achieved by a thin lens.

In Fig. 2(a), we illustrate focusing dynamics of a thick lens for spin excitations with the lattice model Eq. (1) in a parameter regime where the continuum approximation is well justified (see below for details). For an initial Gaussian wave packet with spatial width $\sigma_{0}$, corresponding to a (in the continuum limit) cigar-shaped Wigner function $W(x, p, 0)=(1 / \pi) \exp \left[-\left(x / \sigma_{0}\right)^{2}-\left(\sigma_{0} p\right)^{2}\right]$, the spatial width $\sigma(t)^{2}=\sigma_{0}^{2}\left[\cos ^{2} \omega t+\left(\ell / \sigma_{0}\right)^{4} \sin ^{2} \omega t\right]$ starts oscillating as a function of time [see red line in Fig. 2(a)] and has periodic minima at every quarter of a period $\omega t_{f}=\pi / 2$, where $\sigma_{f} \equiv \sigma\left(t_{f}\right)=\ell^{2} / \sigma_{0}$. The final width in real space (after a quarter of a period) corresponds to the Fourier transform of the initial wave function, i.e., $\psi\left(x, t_{\text {foc }}\right)=$ $\mathcal{F}_{x^{\prime}}\left\{\psi\left(x^{\prime}, 0\right)\right\}\left(x / \ell^{2}\right)$, as for an optical lens. The focusing in real space is illustrated in Fig. 2, where panels 1-4 show the corresponding phase space dynamics of the Wigner function.

Instead of the "always-on" Hamiltonian Eq. (1) of the thick lens, focusing can also be obtained in a pulsed scheme, where the quadratic potential term is switched on for a short time only. This imprints a position-dependent momentum kick $\Delta k a=-2 \phi_{0}\left(n-n_{f}\right)$ (with $\phi_{0}>0$ ) onto the initial wave function via the quadratic phase shift $\hat{U}_{\phi_{0}}=$ $\exp \left[-i \phi_{0} \sum_{n}\left(n-n_{f}\right)^{2} \hat{\sigma}_{z}^{(n)}\right]$, followed by a free evolution of the spin system via Eq. (1) with $v_{0}=0$, as illustrated in Fig. 2(b). This is in analogy with a thin lens in classical optics, where a thin refractive material imprints a positiondependent phase onto the incoming plane wave. The Wigner function in phase space first acquires a momentum kick $p \rightarrow p-2 \phi_{0} x / a^{2}$, followed by free evolution corresponding to a shear motion of the Wigner function, i.e., $W(x, p, t)=W(x-p t / m, p, 0)$, as illustrated in panels 14 of Fig. 2(b). In contrast to the thick lens there is a single focal time $J t_{f}=2\left(\sigma_{0} / a\right)^{4} \phi_{0} /\left[4 \phi_{0}^{2}\left(\sigma_{0} / a\right)^{4}+1\right]\left[\approx 1 /\left(2 \phi_{0}\right)\right.$ for $\sigma_{0} \gg a$ ], where a Gaussian wave function has its minimum width $\sigma_{f}=\sigma_{0} / \sqrt{4 \phi_{0}^{2}\left(\sigma_{0} / a\right)^{4}+1}$.
Figure 2(c) shows the spatial excitation probability for lattice sites around the focus. The green bars correspond to the dynamics illustrated in Fig. 2(a). An excitation initially delocalized over $\sigma_{0} / a=100$ lattice sites gets localized on $\sigma_{f} / a=2.7$ lattice sites using a thick lens including corrections up to sixth order (described in Sec. II B). One can improve the focusing by using multiple pulses or even combining the two different schemes. For example, we can further focus the spin excitation from $\sigma_{0} / a=2.7$ lattice sites to $\sigma_{f} / a=1.2$ lattice sites, shown as the yellow bars in Fig. 2(c), by adjusting the lens strength to the new initial condition.

\section{B. Lattice corrections: Dephasing and Bloch oscillations}

Corrections to the continuum limit become important when the delocalized excitation is focused to a spatial region on the order of the lattice spacing, and the Wigner function extends close to the border of the first Brillouin zone. This happens for sufficiently strong potential in Eq. (1), which leads to aberration and Bloch oscillations due to deviation from a quadratic dispersion relation. In the following, we discuss the limitations this imposes on the achievable final width and show how the effects of the nonquadratic dispersion relation can be compensated using nonparabolic lens potentials.

The main results are summarized in Fig. 3(a), where the numerically obtained final width $\sigma_{f}$ is plotted as a function of the initial width $\sigma_{0}$ and lens strength $v_{0}$ or $\phi_{0}$ for the (i) thick and (ii) thin lens on a lattice, respectively. Simulations have been performed on a 1D chain with $L=10^{6}\left(L=10^{5}\right)$ spins according to Eq. (1) for the thin (thick) lens setup. In contrast to the continuum picture, where a stronger lens leads to a tighter spatial focus and a faster focusing time, the numerical results show that there exist optimal lens potentials (see below) scaling as

$$
v_{\mathrm{opt}} \sim\left(\frac{a}{\sigma_{0}}\right)^{8 / 3} \quad \text { and } \quad \phi_{\mathrm{opt}} \sim\left(\frac{a}{\sigma_{0}}\right)^{4 / 3}
$$

for the thick and thin lens setups, illustrated as black dashed lines in Fig. 3(a). At this optimal lens strength, the final achievable width scales as

$$
\sigma_{f}=a \kappa\left(\frac{\sigma_{0}}{a}\right)^{1 / 3} .
$$

for both the thick and thin lens with $\kappa$ obtained numerically. Focusing works well below this optimal lens strength, in excellent agreement with Figs. 2(a) and 2(b). The scaling of Eq. (3) (black line) is in perfect agreement with the numerically evaluated final width (blue dots) shown in Fig. 3(b) for (i) the thick and (ii) the thin lens with $\kappa=0.68$ and $\kappa=0.80$, respectively. 

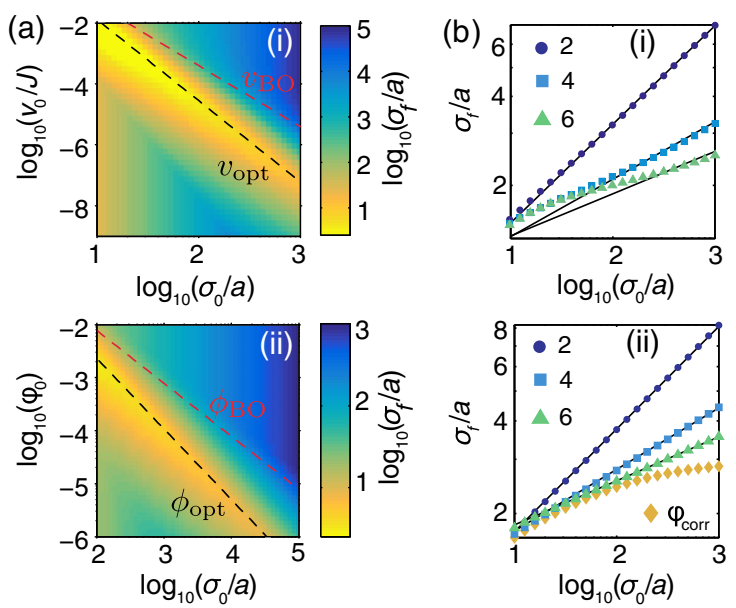

FIG. 3. (a) Final width $\sigma_{f}$ as a function of initial width $\sigma_{0}$ and (i) potential strength $v_{0}$ or (ii) phase $\phi_{0}$ for the (i) thick and (ii) thin lens setup. Dephasing due to the nonquadratic dispersion relation starts to dominate at $v_{0}=v_{\text {opt }}\left(\sigma_{0}\right)$ and $\phi_{0}=\phi_{\text {opt }}\left(\sigma_{0}\right)$ (black dashed line) while Bloch oscillations start at $v_{0}=v_{\mathrm{BO}}\left(\sigma_{0}\right)$ and $\phi_{0}=\phi_{\mathrm{BO}}\left(\sigma_{0}\right)$ (red dashed line) for thick and thin lenses, respectively. (b) Minimum final width $\sigma_{f}$ for the optimized lens strength as a function of initial width for (i) thick and (ii) thin lens setup. Dark blue dots, light blue squares, and green triangles correspond to the numerically obtained final width for a quadratic, quartic, and sixth-order potential, respectively. The black lines are a guide to the eye, showing the respective scalings of Eq. (6).

In the following we discuss the two main effects of the lattice: aberration, giving rise to the optimal lens potentials of Eq. (2), and Bloch oscillations at the edge of the lens shown in Fig. 2(d). We show that the corresponding aberration can be addressed using potentials and pulse shapes that include quartic and higher-order terms.

\section{Aberration}

Deviations from the continuum model can be understood as arising from the nonlinear group velocity $v_{g}(k)=$ $2 J a \sin (k a)$ on the lattice, which implies that wave packets with large momenta propagate more slowly than in the continuum limit, where $v_{g}^{(c)}(k)=2 J a^{2} k$. This results in a path difference $\Delta s(k)=\left[v_{g}^{(c)}(k)-v_{g}(k)\right] t_{f}$ at the focal time $t_{f}$ between the lattice and the continuum model. If this path difference becomes of the order of the final width, i.e., $\Delta s(k) \sim \sigma_{f}$, the continuum approximation breaks down and the wave packet will broaden again. Evaluating this equation at the maximum momentum $1 / \sigma_{f}$ and expanding the path difference up to third order, $\Delta s(k)=-a J t_{f}\left[(k a)^{3} / 3+\mathcal{O}(k a)^{5}\right]$, one obtains Eq. (2) (see also Appendix A). The difference in the group velocities further explains the S-shaped distortion of the Wigner function observed in panels 1-4 of Figs. 2(a) and 2(b) as the nonlinear group velocity induces a nonrigid rotation in phase space.

\section{Bloch oscillations}

At an even larger potential strength, the wings of the wave packet with an extension larger than

$$
\sigma_{\mathrm{BO}}=2 a \sqrt{\frac{J}{v_{0}}}
$$

will undergo Bloch oscillations (see Appendix A), as illustrated in Fig. 2(d). This limits the lens strength to values well below $v_{\mathrm{BO}}=4 J\left(a / \sigma_{0}\right)^{2}$, indicated as a red dashed line in Fig. 3(a). At this critical lens potential the (local) potential gradient $V_{n}^{\prime}=2 v_{0}\left(n-n_{f}\right)$ gives rise to Bloch oscillations with an amplitude $\Delta n=2 J / V_{n}^{\prime}$ and frequency $\omega_{\mathrm{BO}}=V_{n}^{\prime} / 2$ [37]. If this frequency becomes of the order of the focusing time, focusing becomes ineffective. A similar argument for the thin lens yields the maximum pulse strength $\phi_{\mathrm{BO}} \sim a / \sigma_{0}$. In contrast to Bloch oscillations in the thick lens, for the thin lens focusing is limited by phase wraps as the momentum kicks imparted by the pulse extend beyond the first Brillouin zone.

\section{Correction of aberration}

Quartic deviations from the dispersion relation limit the final width of the spin wave to $\sigma_{f} \sim\left(\sigma_{0} / a\right)^{1 / 3}$. Similar to aspherical lenses, the effect of dephasing due to the nonquadratic dispersion relation can be compensated using more general potentials (thick lens) and imprinted phase profiles (thin lens), of the form

$$
V_{n}=\sum_{q=1}^{Q / 2} v_{2 q}\left(n-n_{f}\right)^{2 q},
$$

including additionally quartic $(Q=4)$, sixth $(Q=6)$, or even higher-order terms. Such higher-order terms will accelerate the wings of the wave packet stronger. This allows to compensate the smaller group velocities on the lattice. Using a similar argument as in Appendix A, one can show that for an appropriate choice of $v_{2 q}$ this leads to an improved scaling:

$$
\sigma_{f} \sim\left(\frac{\sigma_{0}}{a}\right)^{1 /(Q+1)} .
$$

Figure 3(b) plots the final width as a function of the initial width for (i) the thin and (ii) the thick lens setup using a lens strength up to $Q=2,4$, and 6 . The numerically obtained final width agrees well with the scaling of Eq. (6).

For the thin lens an optimized form of Eq. (5) can be derived analytically using a semiclassical model with continuous spatial variable $a n \rightarrow x$ and a Bloch band dispersion $\epsilon(k)$ giving rise to a nonlinear group velocity $v_{g}(k)$. Given the imprinted phase profile $\phi(x)$, the initial wave packet receives a position-dependent momentum kick 
$\phi^{\prime}(x) / a$. In order to focus all parts of the wave packet to the focal point $x_{f}=0$ at the same time $t_{f}$, we require $x=v_{g}\left(k\left(\phi^{\prime}\right)\right) t_{f}$, which yields

$$
\phi(x)=-(x / a) \arcsin \left[\phi_{0}(x / a)\right]-\sqrt{\phi_{0}^{2}-(x / a)^{2}},
$$

with $t_{f}=1 /\left(2 J \phi_{0}\right)$. Panel (ii) of Fig. 3(b) shows the final width obtained using Eq. (7), with $x=n a$ on a lattice (yellow diamonds), which shows a clear improvement over the parabolic phase profile. Note that $\phi(x)$ is only real valued up to $|x / a|=\phi_{0}$ due to the maximum group velocity $v_{g}(\pi / 2)=2 J$ on a lattice, since only parts of the wave packets with distance $x<2 J t_{f}$ can be focused within the focusing time.

\section{Multifocal lenses and generation of EPR states}

Instead of the single focus lens, as in Eq. (1), we can employ double-well, or multiwell potentials. The corresponding potentials can be generated as spin-dependent optical potentials, and an array of spin lenses can be realized with large spacing optical lattices. A multifocal lens operating on a single initial delocalized spin excitation will generate a superposition state of excitations at the focal points. For two foci, for example, we can generate an EPR-type state

$$
\hat{S}^{+}|G\rangle \rightarrow\left(\hat{\sigma}_{+}^{\left(n_{1}\right)}+\hat{\sigma}_{+}^{\left(n_{2}\right)}\right)|G\rangle .
$$

Thus, we generate a superposition (EPR state) between spins at lattice site $n_{1}$ and $n_{2}$, as schematically illustrated in panel (ii) of Fig. 1(c).

The time evolution of the corresponding multifocal lens is visualized in panel (ii) of Fig. 1(e). In the upper half plane $\left(y_{n}>0\right)$ we use the $2 \mathrm{D}$ potential $V\left(x_{n}, y_{m}\right)=$ $v_{0}\left[\left(x_{n}-x_{f}\right)^{2}+\left(y_{m}-y_{f}\right)^{2}\right]$ with focal points $\left(x_{f}, y_{f}\right)=$ $(0, \sqrt{2} \times 10) a$, while in the lower half plane $\left(y_{n}<0\right)$ we use $V\left(x_{n}, y_{m}\right)$ with $\left(x_{f}, y_{f}\right)=(0,-\sqrt{2} \times 10) a$. Note that in Fig. 1(e) we rotate the potential by $45 \mathrm{deg}$. The potential strength is optimized to $v_{0}=4.5 \times 10^{-3} \mathrm{~J}$ for an initially symmetric Gaussian wave function with radial spatial width $\sigma_{0}=10 a$ on a 2D lattice with $L_{x}=L_{y}=50$ lattice sites.

\section{Long-range flip-flop interactions}

Implementations of Hamiltonian Eq. (1) with Rydberg atom in optical lattices or strings of trapped ions motivate a model

$$
\hat{H}=-\sum_{n, m} J_{n}\left[\hat{\sigma}_{+}^{(m)} \hat{\sigma}_{-}^{(m+n)}+\text { H.c. }\right]+\sum_{n} V_{n} \hat{\sigma}_{z}^{(n)},
$$

with long-range flip-flop interactions $J_{n}=J_{0} / n^{\alpha}$. In particular, dipolar and van der Waals interactions between
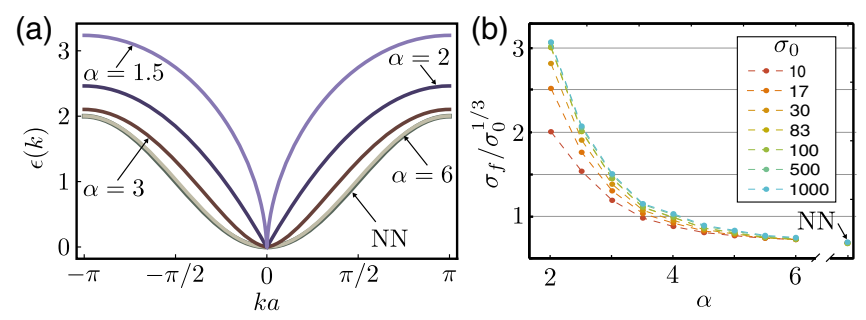

FIG. 4. (a) Dispersion relation $\epsilon_{\alpha}(k)$ for long-range flip-flop interactions (see text) for different exponents $\alpha$ and nearestneighbor (NN) interactions. (b) Rescaled final width $\sigma_{f} / \sigma_{0}^{1 / 3}$ for different exponents $\alpha$ and for different initial conditions $\sigma_{0}$. The data points on the very right correspond to nearest-neighbor interactions.

Rydberg (dressed) atoms allow us to realize $\alpha=3$ and $\alpha=6$ [3], respectively, while $0<\alpha<3$ can be realized with strings of ions [5-8]. The first term of Eq. (9) gives rise to a dispersion relation $\epsilon_{\alpha}(k)=2 \sum_{n}[1-\cos (n k a)] / n^{\alpha}$, as shown in Fig. 4(a). While for $\alpha=6$ the dispersion relation $\epsilon_{6}^{\prime \prime}(0)=\pi^{4} / 90 \approx 1.08$ closely resembles the one of the nearest-neighbor flip-flop interactions of Eq. (1), for $\alpha<3$ the dispersion relation exhibits a kink, e.g., $\epsilon_{2}(k)=$ $(\pi / 2)|k a|-(k a)^{2} / 4$, resulting in a linear group velocity at small momenta with a discontinuity at $k=0$. This leads to strong aberration and inefficient focusing. We note that this can be corrected using an adiabatic lens scheme [36].

Figure 4(b) shows $\kappa \equiv \sigma_{f} /\left(a^{2} \sigma_{0}\right)^{1 / 3}$ [see Eq. (3)] for different realizations of $\alpha$ for the thick lens setups. While for large values of $\alpha$ the scaling of Eq. (3) agrees with the numerically obtained final width, for smaller values the linear dispersion relation leads to strong deviations. The smallest final width (smallest $\kappa$ ) is obtained for large values of $\alpha$ and ultimately with nearest-neighbor interactions, however, $\alpha=6$ almost perfectly resembles nearest-neighbor interactions.

\section{NONLINEAR QUANTUM SPIN LENS: FOCUSING AND SPATIAL SORTING OF MULTIPHOTON STATES}

While the lenses discussed so far are linear lenses operating on single spin excitations, we can also design nonlinear lenses, where the focusing dynamics depend on the number of spin excitations in the medium via spin-spin interactions. Returning to the light-matter interface we discuss at the beginning of Sec. II, we now generalize to an incident multiphoton superposition state $|\mathcal{E}\rangle=$ $\sum_{\nu=0}^{\infty} c_{\nu}|\nu\rangle$. For a write process to atomic quantum memory using a Raman scheme involving a pair of atomic groundstate levels (as described in Ref. [38]), this multiphoton state is mapped to a superposition of (dilute) delocalized spin excitations, $|\mathcal{E}\rangle \rightarrow \sum_{\nu} c_{\nu}\left(\hat{S}^{+}\right)^{\nu}|G\rangle / \sqrt{\nu !}$ (representing hardcore bosons). Repulsive spin-spin interactions, which become relevant during the focusing dynamics when the 
excitation density increases, will map this superposition state to a superposition of spatial spin patterns in an atomic quantum memory. We note that this provides a means of manipulating the individual terms in the superposition state by spatially addressing the atomic spins with a laser. These transformed superposition states of spins can then be mapped back to photons in a defocusing and read operation, providing effective nonlinearities and manipulation of quantum states on the single photon level.

Nonlinear quantum lenses can be implemented by generalizing the Hamiltonian Eq. (1) to

$$
\begin{aligned}
\hat{H}= & -J \sum_{n}\left[\hat{\sigma}_{+}^{(n)} \hat{\sigma}_{-}^{(n+1)}+\text { H.c. }\right]+\sum_{n} V_{n} \hat{\sigma}_{z}^{(n)} \\
& +\sum_{n} \sum_{m} J_{z}^{(m)} \hat{\sigma}_{z}^{(n)} \hat{\sigma}_{z}^{(n+m)},
\end{aligned}
$$

with the last term a long-range $J_{z}^{(m)}=J_{z} / m^{6}$ spin-spin interaction and blockade distance $r_{B}=a\left(J_{z} / J\right)^{1 / 6}$. We emphasize that the spin-spin interactions in Eq. (10) arise naturally in Rydberg (dressed) gases and in trapped ion spin models. Time evolution according to the above Hamiltonian will propagate the initial quantum state to a strongly correlated many-body quantum state,

$$
\sum_{\nu=0}^{\infty} c_{\nu} \frac{1}{\sqrt{\nu !}}\left(\hat{S}^{+}\right)^{\nu}|G\rangle \rightarrow \sum_{\nu=0}^{\infty} c_{\nu}\left|\psi_{\nu}\right\rangle,
$$

with

$$
\left|\psi_{\nu}\right\rangle=\sum_{n_{1}, \ldots, n_{\nu}} \psi_{n_{1}, \ldots, n_{\nu}}^{(\nu)} \hat{\sigma}_{+}^{\left(n_{1}\right)} \cdots \hat{\sigma}_{+}^{\left(n_{\nu}\right)}|G\rangle
$$

and $\psi_{n_{1}, \ldots, n_{\nu}}^{(\nu)}$ the spatial wave functions for $\nu$ spin excitations.

Figure 5 illustrates these focusing dynamics of interacting spins according to Eq. (10) for an initial superposition state consisting of exactly one, two, or three delocalized spin excitations as a function of time. We plot the excitation probability as a function of position in the lattice, $p_{n}^{(\nu)} \equiv$ $\operatorname{tr}\left\{\hat{\sigma}_{+}^{(n)} \hat{\sigma}_{-}^{(n)}\left|\psi_{\nu}\right\rangle\left\langle\psi_{\nu}\right|\right\}$ at lattice site $n$ for $\nu=1,2,3$, which clearly exhibits the spatial mapping and resolution of spin patterns associated with $\left|\psi_{\nu}\right\rangle$ of Eq. (12). This allows us to perform gate operations on spatially localized atoms, e.g., atoms $n= \pm 4$ or $n= \pm 7$, in order to manipulate the $\nu=2$ or $\nu=3$ contribution of the superposition state individually. We note that the small excitation fraction between the peaks, e.g., population of atoms with $n=-1,0,1$ for $\nu=2$ (green bars), can be traced back to states in the initial wave function where two excitations are closer than $r_{B}$. This fraction of states becomes smaller by decreasing the initial excitation density, i.e., increasing the atom number.
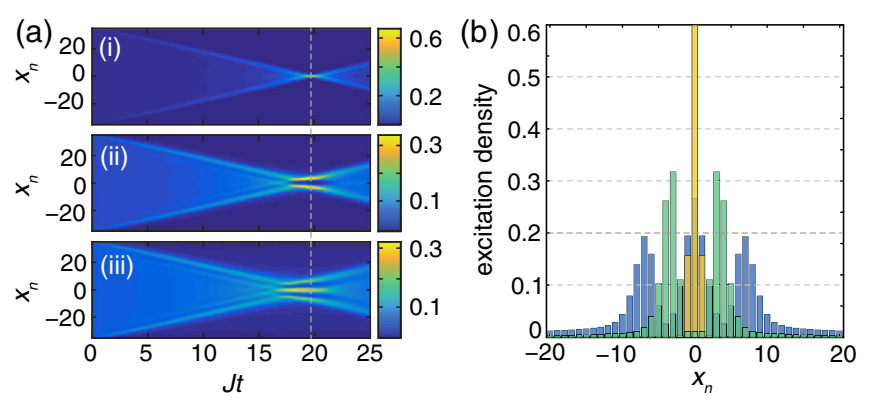

FIG. 5. (a) Time evolution of the excitation density $p_{n}^{(\nu)}(t)$ for (i) $\nu=1$, (ii) $\nu=2$, and (iii) $\nu=3$ initially delocalized spin excitations according to the nonlinear spin-lens Hamiltonian Eq. (10) with interaction range $r_{B}=4.1 a$ and $J_{z}=5 \times 10^{3} J$. (b) Excitation density $p_{n}^{(\nu)}\left(t_{f}\right)$ at the focal time $t_{f}$ for $\nu=1$ (yellow bars), $\nu=2$ (green bars) and $\nu=3$ (blue bars) excitations, demonstrating spatial spin pattern formation depending on the number of excitations $\nu$.

The above can be immediately generalized to higher dimensions. In particular, Fig. 1(e), bottom panel (iii), illustrates focusing of two spin excitations $(\nu=2)$ in 2D. In this case, the repulsive spin-spin interactions give rise to a superposition of states with two excitations separated by a characteristic distance $r_{B}$ around the single-excitation focus forming a ring, reminiscent of a quantum crystal.

\section{IMPLEMENTATION WITH RYDBERG ATOMS IN 2D AND 3D ARRAYS}

The quantum spin lenses we propose in the previous sections can be implemented with atoms stored in optical trap arrays, including large spacing optical lattices and optical tweezers $[26,39,40]$ in $1 \mathrm{D}, 2 \mathrm{D}$, and $3 \mathrm{D}$, or alternatively with trapped ions in 1D [5-8].

Below we describe first a realization of a linear spin-lens Hamiltonians of the type Eq. (1) in 1D, but in particular also in 2D and 3D with alkali Rydberg atoms, where the spin degree of freedom of Sec. II is represented by a pair of levels involving a long-lived atomic hyperfine ground state and a highly excited Rydberg state. As an example, we consider ${ }^{87} \mathrm{Rb}$ atoms and choose $|g\rangle=\mid 5 S_{1 / 2}, F=2$, $\left.m_{F}=2\right\rangle$ as the spin-down and $|s\rangle=\left|n S_{1 / 2}, m_{j}=1 / 2\right\rangle$ as the spin-up state [see Fig. 6(a)]. Note that in this section we denote by $n$ the principal quantum number.

Long-range spin exchange interactions $J\left(r_{i j}\right)=J / r_{i j}^{6}$ between spins $i$ and $j$ in 3D can be achieved by weakly dressing the atomic ground state $|g\rangle$ by admixing with a laser a second Rydberg state $\left|s^{\prime}\right\rangle=\left|n^{\prime} S_{1 / 2}, m_{j}=1 / 2\right\rangle$ with (effective) Rabi frequency $\Omega$ and detuning $\Delta \gg \Omega$. This particular choice of Rydberg states leads to spin couplings $J\left(r_{i j}\right)$, which are isotropic in $3 \mathrm{D}$, i.e., a purely radial dependence as a function of the distance $r_{i j}=\left|\mathbf{r}_{i}-\mathbf{r}_{j}\right|$ for a large range of principal quantum numbers $[41,42]$. We note that, e.g., dipolar exchange interactions can be 
(a)
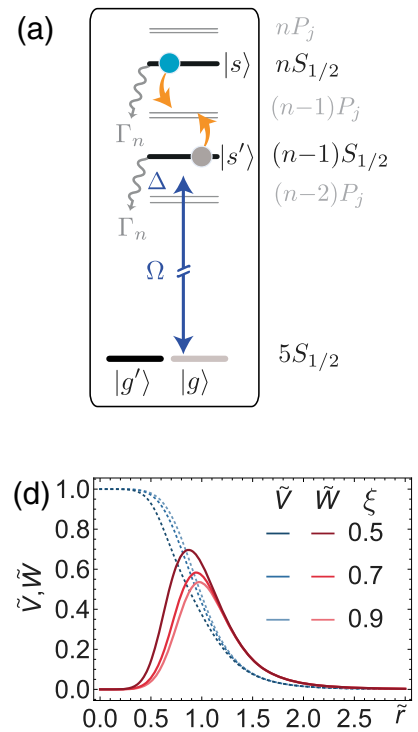
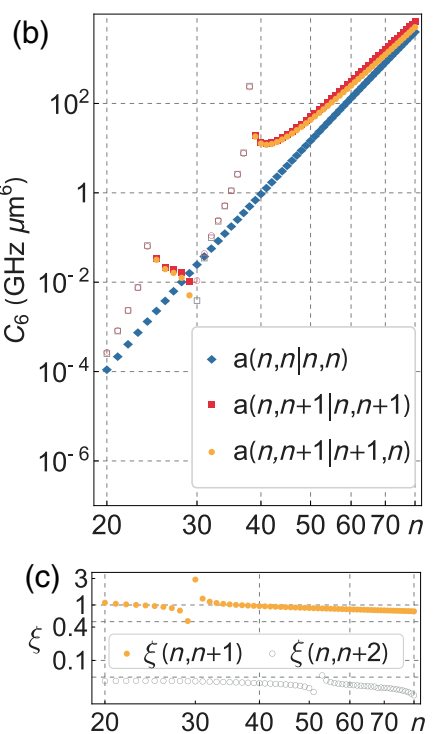

FIG. 6. (a) Atomic level scheme (see text). (b) vdW coefficients between Rydberg $n S_{1 / 2}$ and $n^{\prime} S_{1 / 2}$ states as a function of principal quantum number $n$. Negative values are plotted as outlined markers, positive values as filled. (c) Exchange interaction strength $\xi(n, n+\delta n) \equiv a(n+\delta n \mid n, n+\delta n) /$ $a(n, n+\delta n \mid n, n+\delta n)$ as a function of principal quantum number $n$. (d) Effective interaction potentials of Eq. (15) for $\xi=0.5$, 0.7 , and 0.9 , e.g., corresponding to $n=29,90$, and 27, respectively.

engineered by dressing the ground state with Rydberg $\left|n^{\prime} P_{j}, m_{j}\right\rangle$ states resulting in anisotropic flip-flop interactions of the form $J_{i j} \sim J / r_{i j}^{3}$ [43].

To obtain the desired flip-flop term in Eq. (1) we first consider two atoms and derive an effective Hamiltonian for the dynamics between the dressed ground state and the Rydberg state. We start with a microscopic Hamiltonian, $\hat{H}_{\text {mic }}=\hat{H}_{A}^{(1)}+\hat{H}_{A}^{(2)}+\hat{H}_{\text {int }}$, where the first two terms account for the two driven atoms with $\hat{H}_{A}^{(i)}=$ $-\Delta|s\rangle_{i}\langle s|+(\Omega / 2)| g\rangle_{i}\langle s|+$ H.c., written in a rotating frame. A small magnetic field and a circularly polarized laser beam allow dressing of the ground state with a specific Zeeman sublevel of the Rydberg state.

The key element of the implementation is the van der Waals (vdW) interaction $\hat{H}_{\mathrm{vdW}}$ between the $n S_{1 / 2}$ and $n^{\prime} S_{1 / 2}$ Rydberg states. Choosing two $s$ states ensures that the resulting vdW interactions are isotropic in 3D over a large range of principal quantum numbers $n$ (see Appendix B). The exchange interaction between the degenerate states $\left|n S_{1 / 2}, 1 / 2\right\rangle \otimes\left|n^{\prime} S_{1 / 2}, 1 / 2\right\rangle \quad$ and $\left|n^{\prime} S_{1 / 2}, 1 / 2\right\rangle \otimes\left|n S_{1 / 2}, 1 / 2\right\rangle$ dominantly arises via virtual population of $\left|(n-1) P_{j}, m_{j}\right\rangle \otimes\left|\left(n^{\prime}+1\right) P_{j^{\prime}}, m_{j}^{\prime}\right\rangle$ Rydberg states [see Fig. 6(a)] and strongly depends on $n$ and $n^{\prime}$. As a particular example to demonstrate the tunability of the resulting spin interactions, we discuss the case $n^{\prime}=n-1$ for which the exchange process is maximized [see Fig 6(c)]. The interaction has the structure

$$
\hat{H}_{\mathrm{vdW}}=\frac{1}{r^{6}}\left(\begin{array}{ccc}
c_{11} & 0 & 0 \\
0 & c_{12} & w_{12} \\
0 & w_{12} & c_{12}
\end{array}\right),
$$

written in the basis of states $|s, s\rangle,\left|s, s^{\prime}\right\rangle$, and $\left|s, s^{\prime}\right\rangle$, where we neglect the $\left|s^{\prime}, s^{\prime}\right\rangle$ interactions, since we start initially with only one excitation (i.e., consider a linear lens). The generalized vdW coefficients $c_{i j}=a\left(n_{i}, n_{j} \mid n_{i}, n_{j}\right)$ (diagonal) and $w_{i j}=a\left(n_{i}, n_{j} \mid n_{j}, n_{i}\right)$ (exchange) are shown in Fig. 6(a) and derived in Appendix B. The linear behavior (on the $\log$ scale) of $c_{11}$ shows the typical $n^{11}$ scaling of vdW interactions. The resonances in $c_{12}$ and $w_{12}$ for $n=25$ and $n=40$ stem from vanishingly small energy differences between the states $\left|n P_{3 / 2}, n P_{1 / 2}\right\rangle$ and $\left|n P_{3 / 2}, n P_{3 / 2}\right\rangle$, respectively. Close to one of the Förster resonances diagonal and off-diagonal interactions become approximately equal, $c_{12} \approx w_{12}$.

Adiabatic elimination of $\left|s^{\prime}\right\rangle$ in the limit $\Omega \ll \Delta$ leads to an effective long-range spin model between the dressed ground state $|g\rangle$ and the Rydberg state $|s\rangle$ of the form

$$
\hat{H}_{\mathrm{eff}}=\sum_{i j}\left[V_{s g}^{(i j)} \hat{\sigma}_{g g}^{(i)} \hat{\sigma}_{s s}^{(j)}+\frac{1}{2} W_{s g}^{(i j)} \hat{\sigma}_{g s}^{(i)} \hat{\sigma}_{s g}^{(j)}\right],
$$

with Pauli operators $\hat{\sigma}_{i j}=|i\rangle\langle j|$ and effective laser admixed interactions $V_{s g}^{(i j)}=\Omega^{2} /(4 \Delta) \tilde{V}\left(r_{i j}\right)$ and $W_{s g}^{(i j)}=$ $\Omega^{2} /(2 \Delta) \tilde{W}\left(r_{i j}\right)$ given by

$\tilde{V}=\frac{\tilde{r}^{12}+\tilde{r}^{6}}{\left(\tilde{r}^{6}+1\right)^{2}-\xi^{2}} \quad$ and $\quad \tilde{W}=\frac{\xi \tilde{r}^{6}}{\left(\tilde{r}^{6}+1\right)^{2}-\xi^{2}}$.

Here, $\tilde{r}=\left(|\Delta| / c_{12}\right)^{1 / 6} r(\Delta<0)$ is a dimensionless distance and $\xi=w_{12} / c_{12}$ is the relative exchange strength (see Fig. 6). Note that we drop the ac-Stark shift, which affects all dressed states equally. The potentials are shown in Fig. 6(a) as a function of interatomic distance. As a particular example we consider dressing to the $n^{\prime}=59$ Rydberg state with (two-photon) Rabi frequency $\Omega / 2 \pi=$ $10 \mathrm{MHz}$ and detuning $\Delta / 2 \pi=-20 \mathrm{MHz}$. The lattice constant $a$ is adjusted to the maximum of $\tilde{W}$ which results in $J / 2 \pi=0.36 \mathrm{MHz}$. For a delocalized spin excitation with initial width $\sigma_{0}=100 a$ the focusing time is $t_{f}=5 \mu \mathrm{s}$ which increases linearly with the initial width $\sigma_{0}$. This compares well with the lifetime of the state $|s\rangle=\left|60 S_{1 / 2}, m_{j}=1 / 2\right\rangle$, with $\tau_{60 S}=252 \mu$ s [44], resulting in $t_{f} / \tau_{60 S} \approx 0.02$.

The single particle potential of Eq. (9) can be realized via an ac-Stark shift on the ground state. As a first approximation, a quadratic potential can be applied using a wide Gaussian beam with a beam waist on the order of the system size. Higher-order corrections can be implemented with more sophisticated beam-shaping techniques using, for instance, digital micromirror devices. 
Instead of the spin models with atomic ground and Rydberg states representing a spin-1/2 system, one can also employ dressing schemes, where spin is represented by a pair of long-lived and trapped atomic ground states, and spin hopping and interaction terms are obtained by admixing Rydberg interactions with laser light [27,28]. Such schemes may be convenient for the nonlinear lenses we describe in Sec. III.

\section{EFFECTS OF DISORDER ON FOCUSING DYNAMICS}

In this section, we analyze the robustness of the focusing dynamics against two types of static disorder in the spin lattice: (i) holes in the lattice and (ii) static fluctuations of the atomic position resulting in a random distribution of long-range spin couplings $V_{s g}^{(i j)}$ and $W_{s g}^{(i j)}$ in Eq. (14).

\section{A. Nonunity filling}

Missing atoms in the lattice may arise due to imperfect loading or when a previously focused spin wave is stored in a different hyperfine ground state. In addition to being excluded from the hopping matrix $W_{s g}$, each hole is surrounded by an effective potential due to the modification of $V_{s g}$ in Eq. (14). We numerically investigate the effect of randomly distributed holes in both one and two dimensions. Figure 7(a) shows the spin-excitation probability within a radius $3 a$ around the focus of the lens $P_{\text {foc }}$ for a 1D and 2D spin lattice of $N=70$ and $N=70 \times 70$ sites, respectively. Each data point is obtained by averaging over 1000 random hole realizations for the 1D example (400 realizations for 2D), starting with a Gaussian wave function with initial width $\sigma_{0}=14 a$. The width of the statistical
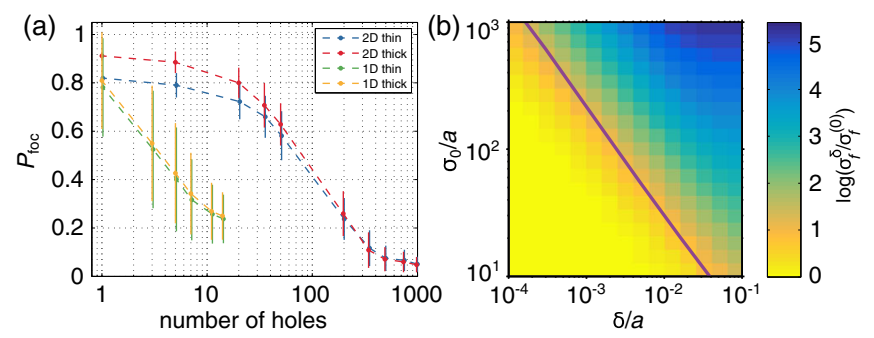

FIG. 7. (a) Integrated probability $P_{\text {foc }}$ to find the focused spin wave inside a circle of radius 3 around the set focal point as a function of the number of holes in the lattice. (b) Final width $\sigma_{f}^{\delta}$ of a spin wave in a disordered system compared to the final width $\sigma_{f}^{(0)}$ without disorder as a function of the initial width $\sigma_{0}$ and the disorder strength $\delta$. The results are obtained for a thick lens including corrections up to sixth order (described in Sec. II B), where the potential strength and the focusing time are chosen to minimize $\sigma_{f}^{(0)}$ for each value of $\sigma_{0}$. The line represents $\delta \sim 1 / t_{\text {foc }}$, indicating the breakdown of focusing due to disorder (details in text).

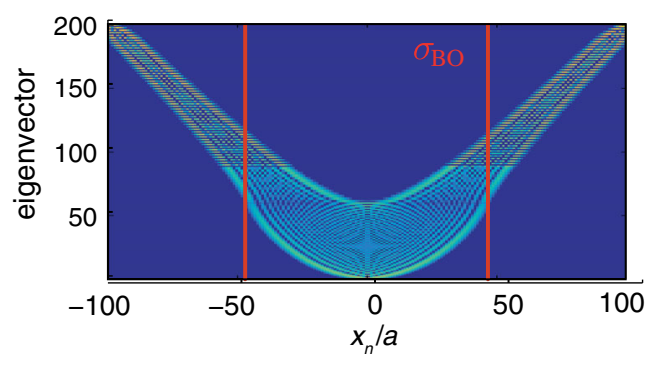

FIG. 8. Bloch oscillations: eigenfunctions of Hamiltonian Eq. (1) illustrating Wannier-Stark localized eigenfunctions at the edge of the lens (see text).

distribution of the final probability is indicated by the error bars.

For the 1D case, a single hole already has a significant detrimental effect on the final wave packet, which we attribute to the fact that $W_{s g} \sim 1 / r^{6}$ closely resembles nearest-neighbor hopping. By contrast, in 2D the focusing scheme is almost unaffected by a small number of holes $(\lesssim 10)$, as the spin wave can flow around the holes. This is further illustrated in panel (i) of Fig. 1(e) as a sequence of snapshots showing the 2D lattice of spins as a function of time. We expect the focusing scheme to be even more robust in three dimensions, as there are more paths to avoid the holes.

\section{B. Static disorder in atomic positions}

As a second form of disorder we analyze the effect of fluctuations of the long-range spin couplings $V_{s g}$ and $W_{s g}$. Such models have previously been discussed in the context of Rydberg atoms trapped in optical tweezers [45]. We assume that the position of the $n$th atom is given by $\mathbf{r}_{n}=$ $\mathbf{r}_{n}^{(0)}+\mathbf{d}_{n}$, with $\mathbf{r}_{n}^{(0)}$ the position on a regular lattice and the displacement $\mathbf{d}_{n} \sim \mathcal{N}\left(0, \delta^{2}\right)$ drawn from a normal distribution with zero mean and standard deviation $\delta$. This results in a change of the interatomic separation $\mathbf{r}_{n}-\mathbf{r}_{m}=$ $\mathbf{r}_{n m}^{(0)}+\mathbf{d}_{n m}$, in turn modifying the diagonal and hopping potentials of Eq. (14) to $W_{s g}\left(r_{i j}\right) \approx W_{s g}\left(r_{i j}^{(0)}\right)+$ $d_{i j} W_{s g}^{\prime}\left(r_{i j}^{(0)}\right)$ and $V_{s g}\left(r_{i j}\right) \approx V_{s g}\left(r_{i j}^{(0)}\right)+d_{i j} V_{s g}^{\prime}\left(r_{i j}^{(0)}\right)$. We note that the first-order term in the expansion of $W_{s g}$ may vanish for certain separations, e.g., nearest neighbors, if the maximum of the interaction potential $\tilde{W}$ is commensurate with the lattice. However, the first-order term will be present for all other separations, as well as in the expansion of $V_{s g}$, which exhibits no maximum or minimum as a function of distance (see Fig. 6).

Disorder tends to localize the eigenstates of the system and thus prevents focusing when the localization length is smaller than the initial width of the wave packet [46]. However, the focusing fidelity may be significantly reduced even if the localization length is large. To quantify the role of disorder, we estimate the energy broadening of plane 

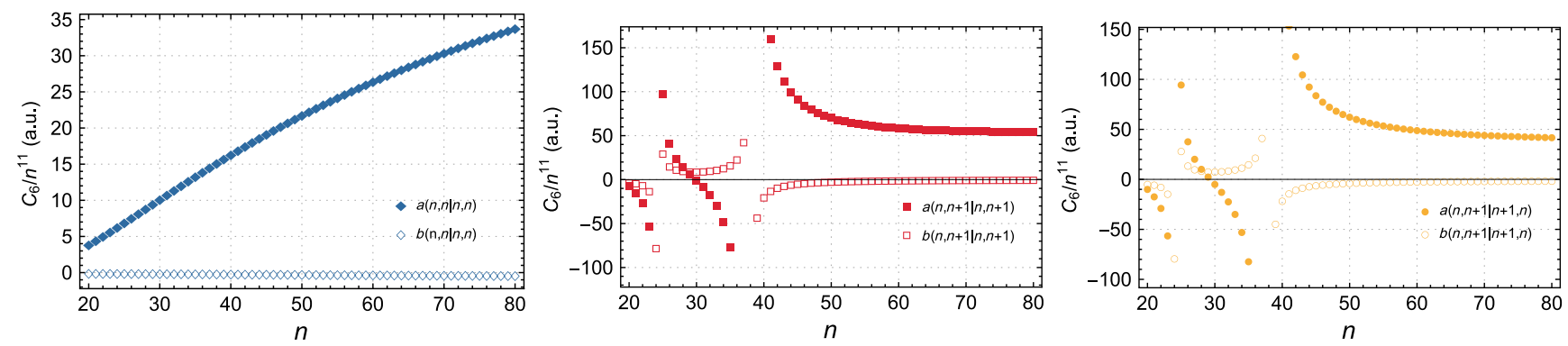

FIG. 9. Generalized vdW coefficients of Eq. (B4) as a function of principal quantum number $n$ for the $n S_{1 / 2}$ and $(n+1) S_{1 / 2}$ Rydberg states.

wave states due to position disorder. In a regular lattice, plane wave states with momentum $k$ are energy eigenstates following the dispersion relation $\epsilon(k)$. In the presence of weak disorder, states with similar momenta are coupled together such that the energy of a plane wave acquires an uncertainty on the order of $\Delta \epsilon(k)=\sqrt{\left\langle k\left|\left(\hat{H}-\hat{H}_{0}\right)^{2}\right| k\right\rangle}$, where $\hat{H}$ and $\hat{H}_{0}$ denote the Hamiltonian of the disordered and the clean system, respectively. Since our scheme sensitively relies on the interference between different momentum states, we expect that focusing ceases to be effective when the focusing time exceeds $t_{\text {foc }} \gtrsim 1 / \Delta \epsilon$. Given that $\Delta \epsilon \sim \delta$ to lowest order in $\delta$, this suggests that there exists a critical disorder strength $\delta_{c} \sim 1 / t_{\text {foc }}$ above which the disorder strongly affects the focusing dynamics. Indeed, this simple argument correctly predicts the breakdown of focusing as demonstrated in Fig. 7(b). We note that we numerically verify that the argument applies equally well to the thin lens.

\section{CONCLUSION AND OUTLOOK}

In this work we show that lenses for spin qubits can be designed for atomic lattice gases, allowing focusing of delocalized spin excitations in quench dynamics to essentially single atoms. In addition, we provide an implementation of a spin lens based on Rydberg-dressed spin-spin interactions. The present work defines a novel light-matter interface, where incoming photons are stored in delocalized atomic excitations in an atomic medium, with spin focusing providing the link and mapping to storage of qubits in single atoms. We note that existing experimental setups with Rydberg atoms [21-24] enabling the physical realization of 1D and 2D $X Y$-spin models can provide first proofof-principle experiments: here, a single delocalized spin excitation as initial condition could be generated using the Rydberg-blockade mechanism in an atomic lattice $[39,43,47]$, and with focusing dynamics implemented as we describe in the present work. This scenario could also be demonstrated with the spin models realized with trapped ions $[7,8]$. Finally, we expect that optimal coherent control techniques both for spatial and temporal model parameters should allow for significant improvement of spin lenses [48].

\section{ACKNOWLEDGMENTS}

We acknowledge discussions with M. Heyl, B. Lanyon and UQUAM partners C. Gross and I. Bloch. Work at Innsbruck is supported by the Austrian Science Fund SFB FoQuS (FWF Project No. F4016-N23), the European Research Council (ERC) Synergy Grant UQUAM, EU H2020 FET Proactive project RySQ, and Scalable Ion-Trap Quantum Network (SciNet). Work at Harvard is supported through NSF, CUA, AFOSR Muri, and the Vannevar Bush Faculty Fellowship. A. W. G. acknowledges funding from the National Research Foundation and the Ministry of Education of Singapore. S. C. acknowledges support from Kwanjeong Educational Foundation. H. P. is supported by the NSF through a grant for the ITAMP at Harvard University and the Smithsonian Astrophysical Observatory.

\section{APPENDIX A: LATTICE CORRECTIONS}

In this Appendix, we discuss dephasing and Bloch oscillations on the lattice and derive Eq. (2) for the optimal lens strength and Eq. (3) for the scaling of the final width.

\section{Dephasing}

The optimal potential strengths $v_{c}$ and optimal pulse strength $\phi_{c}$ of Eq. (2) for the thick and thin lens, respectively, can be derived from the Bloch-band dispersion relation $\epsilon(k)=2 J[1-\cos (k a)]$ and its deviations from the quadratic dispersion relation $\epsilon(k)-J(k a)^{2} \approx J(k a)^{4} / 12+$ $\mathcal{O}(k a)^{6}$. If these deviations become of the order of the inverse focusing time, i.e., $J(k a)^{4} / 12 \sim t_{\text {foc }}^{-1}\left(v_{0}\right)$, then plane wave eigenstates will dephase during the focusing dynamics. This happens for parts of the Wigner function exceeding a critical momentum $k_{c} a=\left[2304 v_{0} /\left(\pi^{2} J\right)\right]^{1 / 8}$ for the thick lens and $k_{c} a=\left(24 \phi_{0}\right)^{1 / 4}$ for the thin lens setup. During focusing the distribution of populated momentum states will become broader with the largest width in momentum space, i.e., $k_{f} \sim 1 / \sigma_{f}$, at the focusing time. This limits the minimum final width and restricts the lens potential to values below $v_{c}$ for the thick lens, as well as the critical pulse strength $\phi_{c}$ for the thin lens. 


\section{Bloch oscillations}

The onset of Bloch oscillations at $\sigma_{\mathrm{BO}}$ of Eq. (4) and the corresponding critical potential strength $v_{\mathrm{BO}}$ can be understood in a semiclassical model for a particle in a quadratic potential with a Bloch-band dispersion relation, following the (semiclassical) equations of motion $\dot{x}=2 J a \sin (k a)$ and $\dot{k}=-2 v_{0} x$ for position and momentum, respectively. These equations are equivalent to a motion of a classical particle with a quadratic dispersion relation in a modified potential $\quad V_{\mathrm{BO}}(x)=\left[\left(2 v_{0}^{2} x_{0}^{2}-4 v_{0} J\right) a^{2} x^{2}-\left(v_{0}^{2} a^{2}\right) x^{4}\right] / 2$. Depending on its initial position $x_{0}$ being smaller or larger than $\sigma_{\mathrm{BO}}$, the particle will experience either a single-well or a double-well potential. Figure 8 shows the corresponding eigenfunctions of the lattice Hamiltonian Eq. (1). Eigenfunctions that have an extension less than $\sigma_{\mathrm{BO}}$ are well described by discretized harmonic oscillator eigenfunctions centered around the origin, while eigenfunctions with an extension larger than $\sigma_{\mathrm{BO}}$ start to get localized at the minima of the double wells of $V_{\mathrm{BO}}(x)$. Thus, Bloch oscillations start to dominate the focusing dynamics at a critical potential strength, $v_{\mathrm{BO}}=4 J\left(a / \sigma_{0}\right)^{2}$, which is indicated as the red dashed line in Fig. 3(a).

\section{APPENDIX B: RYDBERG INTERACTION BETWEEN ${ }^{87}$ Rb ATOMS IN $n_{1} S_{1 / 2}$ AND $n_{2} S_{1 / 2}$ RYDBERG STATES}

For distances large enough, such that the dipole interaction matrix element between two $S$ states and two $P$ states is larger than the energy difference $\Delta_{F}$ between these pair states, i.e., $V_{\text {dip }}>\Delta_{F}$, we can treat vdW interactions perturbatively. The vdW interaction Hamiltonian between $n_{1} S_{1 / 2}$ and $n_{2} S_{1 / 2}$ Rydberg states can be described by a $16 \times 16$ matrix of the form

$$
H_{\mathrm{vdW}}=\left(\begin{array}{cccc}
M(n, n \mid n, n) & 0 & 0 & 0 \\
0 & M\left(n, n^{\prime} \mid n, n^{\prime}\right) & M\left(n, n^{\prime} \mid n^{\prime}, n\right) & 0 \\
0 & M\left(n^{\prime}, n \mid n^{\prime}, n\right) & M\left(n^{\prime}, n \mid n^{\prime}, n\right) & 0 \\
0 & 0 & 0 & M\left(n^{\prime}, n^{\prime} \mid n^{\prime}, n^{\prime}\right)
\end{array}\right) .
$$

The vdW coefficients are given by

$$
M\left(n_{1}, n_{2} \mid n_{3}, n_{4}\right)=\left\langle n_{1} S_{1 / 2} m_{1}, n_{2} S_{1 / 2} m_{2}\left|H_{\mathrm{vdW}}\right| n_{3} S_{1 / 2} m_{3}, n_{4} S_{1 / 2} m_{4}\right\rangle
$$

which is a $4 \times 4$ matrix in the subspace of Zeeman levels $m= \pm 1 / 2$. The vdW interaction operator

$$
H_{\mathrm{vdW}}=\sum_{n_{\alpha}, j_{\alpha}, m_{\alpha}} \sum_{n_{\beta}, j_{\beta}, m_{\beta}} \frac{V_{\mathrm{dd}}\left|n_{\alpha} P_{j_{\alpha}} m_{\alpha}, n_{\beta} P_{j_{\beta}} m_{\beta}\right\rangle\left\langle n_{\alpha} P_{j_{\alpha}} m_{\alpha}, n_{\beta} P_{j_{\beta}} m_{\beta}\right| V_{\mathrm{dd}}}{E_{n_{1}}+E_{n_{2}}-E_{n_{\alpha}}-E_{n_{\beta}}},
$$

couples $S$ states with energy $E_{n_{1}}$ and $E_{n_{2}}$ to intermediate $P$ states with energies $E_{n_{\alpha}}$ and $E_{n_{\beta}}$ via dipole-dipole interactions

$$
V_{\mathrm{dd}}(\mathbf{r})=-\sqrt{\frac{24 \pi}{5}} \frac{1}{r^{3}} \sum_{\mu, \nu} C_{\mu, \nu ; \mu+\nu}^{1,1 ; 2} Y_{2}^{\mu+\nu}(\vartheta, \varphi)^{*} d_{\mu} d_{\nu} .
$$

Here, $\mathbf{d}$ is the atomic dipole operator and $\mathbf{r}=(r, \vartheta, \varphi)$ is the vector between the two atoms in spherical coordinates. With $d_{\mu}$ we denote the $\mu$ th spherical components $(\mu, \nu \in\{-1,0,1\})$ of the atomic dipole operator, $C_{m_{1}, m_{2} ; M}^{j_{1}, j_{2} ; J}$ are Clebsch-Gordan coefficients, and $Y_{l}^{m}$ are spherical harmonics. Using the Wigner-Eckart theorem the vdW interactions can be split up in an angular and radial part,

$$
M\left(n_{1}, n_{2} \mid n_{3}, n_{4}\right)=\frac{1}{r^{6}}\left[a\left(n_{1}, n_{2} \mid n_{3}, n_{4}\right) \mathbb{1}_{4}+b\left(n_{1}, n_{2} \mid n_{3}, n_{4}\right) \mathcal{D}_{0}\right],
$$

with generalized isotropic and anisotropic vdW coefficients

$$
a=\frac{1}{81}\left[7 C_{6}^{(1)}+25 C_{6}^{(2)}+11\left(C_{6}^{(3)}+C_{6}^{(4)}\right)\right], \quad b=\frac{1}{27}\left[C_{6}^{(3)}+C_{6}^{(4)}-C_{6}^{(1)}-C_{6}^{(2)}\right],
$$

and $\mathbb{1}_{4}$ the $4 \times 4$ identity matrix, and 


$$
\mathcal{D}_{0}=\left(\begin{array}{cccc}
\cos (2 \vartheta) & e^{-i \varphi} \sin (2 \vartheta) & e^{-i \varphi} \sin (2 \vartheta) & 2 e^{-2 i \varphi} \sin ^{2}(\vartheta) \\
e^{i \varphi} \sin (2 \vartheta) & \frac{2}{3}-\cos (2 \vartheta) & -\cos (2 \vartheta)-\frac{5}{3} & -e^{-i \varphi} \sin (2 \vartheta) \\
e^{i \varphi} \sin (2 \vartheta) & -\cos (2 \vartheta)-\frac{5}{3} & \frac{2}{3}-\cos (2 \vartheta) & -e^{-i \varphi} \sin (2 \vartheta) \\
2 e^{2 i \varphi} \sin ^{2}(\vartheta) & -e^{i \varphi} \sin (2 \vartheta) & -e^{i \varphi} \sin (2 \vartheta) & \cos (2 \vartheta)
\end{array}\right)
$$

written in the basis $\left\{\left|-\frac{1}{2}-\frac{1}{2}\right\rangle,\left|-\frac{1}{2} \frac{1}{2}\right\rangle,\left|\frac{1}{2}-\frac{1}{2}\right\rangle,\left|\frac{1}{2} \frac{1}{2}\right\rangle\right\}$ of Zeeman states in the $j=1 / 2$ Rydberg manifold and accounting for the anisotropy and mixing between the Zeeman sublevels. With $C_{6}^{(\nu)}$ we denote the radial part of the matrix elements

$$
C_{6}^{(\nu)}\left(n_{1}, n_{2} \mid n_{3}, n_{4}\right)=\sum_{n_{\alpha}, n_{\beta}} \frac{\mathcal{R}_{1}^{\alpha} \mathcal{R}_{2}^{\beta} \mathcal{R}_{3}^{\alpha} \mathcal{R}_{4}^{\beta}}{\delta_{\alpha \beta}}
$$

which accounts for the overall strength of the interaction and is independent of the magnetic quantum numbers. Here, $\mathcal{R}_{i}^{k}=\int d r r^{2} \psi_{n_{i}, \ell_{i}, j_{i}}(r)^{*} r \psi_{n_{k}, \ell_{k}, j_{k}}(r)$ is the radial integral and $\nu$ accounts for the four channels to intermediate $P_{j}$ states.

Figure 9 shows the numerically calculated $a$ and $b$ coefficients corresponding to the different blocks in Eq. (B1) as a function of the principal quantum number $n$. Both $a$ and $b$ show two Förster resonances around $n \approx 24$ and $n \approx 38$ where the channels to $\left\{n P_{3 / 2}, n P_{1 / 2}\right\}$ and $\left\{n P_{3 / 2}, n P_{3 / 2}\right\}$ states become close in energy, respectively.
Apart from these resonances the anisotropy coefficient $b$ is several orders smaller than the diagonal coefficient $a$, which allows us to safely neglect mixing of Zeeman sublevels and results in an almost perfect isotropic interaction.

For two atoms initially in the $\left|n_{1} S_{1 / 2}, 1 / 2\right\rangle$ and $\left|n_{2} S_{1 / 2}, 1 / 2\right\rangle$ states, the dynamics can be restricted to the four $S$ states $S_{1 / 2}$ states with principal quantum numbers $\left|n_{1}, n_{1}\right\rangle,\left|n_{1}, n_{2}\right\rangle, \quad\left|n_{2}, n_{1}\right\rangle$, and $\left|n_{2}, n_{2}\right\rangle$ and magnetic quantum number $1 / 2$. The corresponding Hamiltonian restricted to this basis has the form

$$
H_{\mathrm{vdW}}=\frac{1}{r^{6}}\left(\begin{array}{cccc}
a\left(n_{1}, n_{1} \mid n_{1}, n_{1}\right) & 0 & 0 & 0 \\
0 & a\left(n_{1}, n_{2} \mid n_{1}, n_{2}\right) & a\left(n_{1}, n_{2} \mid n_{2}, n_{1}\right) & 0 \\
0 & a\left(n_{2}, n_{1} \mid n_{2}, n_{1}\right) & a\left(n_{2}, n_{1} \mid n_{2}, n_{1}\right) & 0 \\
0 & 0 & 0 & a\left(n^{\prime}, n^{\prime} \mid n^{\prime}, n^{\prime}\right)
\end{array}\right),
$$

with $a$ denoted as $C_{6}$ in the main text and plotted in Fig. 6(a) as a function of the principal quantum number $n$.

For the long-range Hamiltonian of Eq. (14) [cf. Fig. 6(c)] next-nearest-neighbor hopping is around $10 \%$ of the nearest-neighbor hopping element. In Fig. 4, we compare the performance of a spin lens implemented with the potentials arising from the Rydberg interactions, compared to the case with ideal nearest-neighbor hopping. Our numerical results indicate that long-range hopping terms slightly increase the speed of the scheme and decrease the final width.

[1] T. D. Ladd, F. Jelezko, R. Laflamme, Y. Nakamura, C. Monroe, and J. L. O'Brien, Quantum Computers, Nature (London) 464, 45 (2010).

[2] C. Gardiner and P. Zoller, in The Quantum World of Ultra-Cold Atoms and Light Book II: The Physics of
Quantum-Optical Devices (World Scientific, Singapore, 2015) pp. 1-524.

[3] M. Saffman, T. G. Walker, and K. Mølmer, Quantum Information with Rydberg Atoms, Rev. Mod. Phys. 82, 2313 (2010).

[4] I. Bloch, J. Dalibard, and S. Nascimbene, Quantum Simulations with Ultracold Quantum Gases, Nat. Phys. 8, 267 (2012).

[5] P. Schindler, D. Nigg, T. Monz, J. T. Barreiro, E. Martinez, S. X. Wang, S. Quint, M. F. Brandl, V. Nebendahl, C. F. Roos et al., A Quantum Information Processor with Trapped Ions, New J. Phys. 15, 123012 (2013).

[6] S. Debnath, N. M. Linke, C. Figgatt, K. A. Landsman, K. Wright, and C. Monroe, Demonstration of a Small Programmable Quantum Computer with Atomic Qubits, Nature (London) 536, 63 (2016).

[7] P. Jurcevic, B. P. Lanyon, P. Hauke, C. Hempel, P. Zoller, R. Blatt, and C.F. Roos, Quasiparticle Engineering and Entanglement Propagation in a Quantum Many-Body System, Nature (London) 511, 202 (2014). 
[8] P. Richerme, Z.-X. Gong, A. Lee, C. Senko, J. Smith, M. Foss-Feig, S. Michalakis, A. V. Gorshkov, and C. Monroe, Non-Local Propagation of Correlations in Quantum Systems with Long-Range Interactions, Nature (London) $\mathbf{5 1 1}$, 198 (2014).

[9] A. Reiserer and G. Rempe, Cavity-Based Quantum Networks with Single Atoms and Optical Photons, Rev. Mod. Phys. 87, 1379 (2015).

[10] H. Jeff Kimble, The Quantum Internet, Nature (London) 453, 1023 (2008).

[11] T. E. Northup and R. Blatt, Quantum Information Transfer Using Photons, Nat. Photonics 8, 356 (2014).

[12] D. Hucul, I. V. Inlek, G. Vittorini, C. Crocker, S. Debnath, S. M. Clark, and C. Monroe, Modular Entanglement of Atomic Qubits Using Photons and Phonons, Nat. Phys. 11, 37 (2015).

[13] M. D. Lukin, Colloquium: Trapping and Manipulating Photon States in Atomic Ensembles, Rev. Mod. Phys. 75, 457 (2003).

[14] K. Hammerer, A. S. Sørensen, and E. S. Polzik, Quantum Interface between Light and Atomic Ensembles, Rev. Mod. Phys. 82, 1041 (2010).

[15] M. D. Eisaman, A. André, F. Massou, M. Fleischhauer, A. S. Zibrov, and M. D. Lukin, Electromagnetically Induced Transparency with Tunable Single-Photon Pulses, Nature (London) 438, 837 (2005).

[16] L. Lamata, D. R. Leibrandt, I. L. Chuang, J. I. Cirac, M. D. Lukin, V. Vuletić, and S. F. Yelin, Ion Crystal Transducer for Strong Coupling between Single Ions and Single Photons, Phys. Rev. Lett. 107, 030501 (2011).

[17] T. Peyronel, O. Firstenberg, Q.-Y. Liang, S. Hofferberth, A. V. Gorshkov, T. Pohl, M. D. Lukin, and V. Vuletić, Quantum Nonlinear Optics with Single Photons Enabled by Strongly Interacting Atoms, Nature (London) 488, 57 (2012).

[18] G. Heinze, C. Hubrich, and T. Halfmann, Stopped Light and Image Storage by Electromagnetically Induced Transparency Up to the Regime of One Minute, Phys. Rev. Lett. 111, 033601 (2013).

[19] D. Maxwell, D. J. Szwer, D. Paredes-Barato, H. Busche, J. D. Pritchard, A. Gauguet, K. J. Weatherill, M. P. A. Jones, and C. S. Adams, Storage and Control of Optical Photons Using Rydberg Polaritons, Phys. Rev. Lett. 110, 103001 (2013).

[20] S. Bose, Quantum Communication through Spin Chain Dynamics: An Introductory Overview, Contemp. Phys. 48, 13 (2007).

[21] K. M. Maller, M. T. Lichtman, T. Xia, Y. Sun, M. J. Piotrowicz, A. W. Carr, L. Isenhower, and M. Saffman, Rydberg-Blockade Controlled-NOT Gate and Entanglement in a Two-Dimensional Array of Neutral-Atom Qubits, Phys. Rev. A 92, 022336 (2015).

[22] H. Labuhn, D. Barredo, S. Ravets, S. de Léséleuc, T. Macrì, T. Lahaye, and A. Browaeys, Tunable Two-Dimensional Arrays of Single Rydberg Atoms for Realizing Quantum Ising Models, Nature (London) 534, 667 (2016).

[23] J. Zeiher, R. van Bijnen, P. Schausz, S. Hild, J.-y. Choi, T. Pohl, I. Bloch, and C. Gross, Many-Body Interferometry of a Rydberg-Dressed Spin Lattice, Nat. Phys. 12, 1095 (2016).
[24] Y.-Y. Jau, A. M. Hankin, T. Keating, I. H. Deutsch, and G. W. Biedermann, Entangling Atomic Spins with a RydbergDressed Spin-Flip Blockade, Nat. Phys. 12, 71 (2016).

[25] N. Henkel, R. Nath, and T. Pohl, Three-Dimensional Roton Excitations and Supersolid Formation in Rydberg-Excited Bose-Einstein Condensates, Phys. Rev. Lett. 104, 195302 (2010).

[26] G. Pupillo, A. Micheli, M. Boninsegni, I. Lesanovsky, and P. Zoller, Strongly Correlated Gases of Rydberg-Dressed Atoms: Quantum and Classical Dynamics, Phys. Rev. Lett. 104, 223002 (2010).

[27] A. W. Glaetzle, M. Dalmonte, R. Nath, C. Gross, I. Bloch, and P. Zoller, Designing Frustrated Quantum Magnets with Laser-Dressed Rydberg Atoms, Phys. Rev. Lett. 114, 173002 (2015).

[28] R. M. W. van Bijnen and T. Pohl, Quantum Magnetism and Topological Ordering via Rydberg Dressing near Förster Resonances, Phys. Rev. Lett. 114, 243002 (2015).

[29] I. Novikova, A. V. Gorshkov, D. F. Phillips, A. S. Sørensen, M. D. Lukin, and R. L. Walsworth, Optimal Control of Light Pulse Storage and Retrieval, Phys. Rev. Lett. 98, 243602 (2007).

[30] A. V. Gorshkov, A. André, M. Fleischhauer, A. S. Sørensen, and M. D. Lukin, Universal Approach to Optimal Photon Storage in Atomic Media, Phys. Rev. Lett. 98, 123601 (2007).

[31] Sequential mapping of qubits requires transfer of qubits stored in the excited states $|e\rangle$ to another excited state $\left|e^{\prime}\right\rangle$ to hide these qubits from the focusing dynamics of the following qubits. Note that these previously stored qubits appear as holes (defects) in the focusing dynamics of the consecutive qubits, as discussed in Sec. V.

[32] A. W. Lohmann, Image Rotation, Wigner Rotation, and the Fractional Fourier Transform, J. Opt. Soc. Am. A 10, 2181 (1993).

[33] W. P. Schleich, Quantum Optics in Phase Space (Wiley, New York, 2015).

[34] M. V. Berry, Semi-Classical Mechanics in Phase Space: A Study of Wigner's Function, Phil. Trans. R. Soc. A 287, 237 (1977).

[35] J. P. Bizarro, Weyl-Wigner Formalism for Rotation-Angle and Angular-Momentum Variables in Quantum Mechanics, Phys. Rev. A 49, 3255 (1994).

[36] In contrast to focusing with quench dynamics in a HO, as described above, one could also localize the wave function in an adiabatic ramp of the harmonic oscillator, i.e., by sweeping $\omega \rightarrow \omega_{f}$. An initial Gaussian wave packet, which is matched to represent the $\mathrm{HO}$ ground state with $\omega$ with width $\ell$ would then be mapped to the final ground state with width $\ell_{f} \equiv \sqrt{\hbar / m \omega_{f}}$, with time required $\gg 1 / \omega$. Quench dynamics discussed in this paper results in fast focusing $t_{f} \sim 1 / \omega$, and does not require good knowledge of the initial wave function or control over the applied trapping potential to match the initial wave packet to the $\mathrm{HO}$ ground state. In contrast, an adiabatic scheme can be expected to be more robust against imperfect parameters in Hamiltonian Eq. (1).

[37] M. B. Dahan, E. Peik, J. Reichel, Y. Castin, and C. Salomon, Bloch Oscillations of Atoms in an Optical Potential, Phys. Rev. Lett. 76, 4508 (1996). 
[38] M. Fleischhauer and M. D. Lukin, Quantum Memory for Photons: Dark-State Polaritons, Phys. Rev. A 65, 022314 (2002).

[39] M. Endres, H. Bernien, A. Keesling, H. Levine, E. R. Anschuetz, A. Krajenbrink, C. Senko, V. Vuletic, M. Greiner, and M. D. Lukin, Cold Matter Assembled Atomby-Atom, arXiv:1607.03044.

[40] D. Barredo, S. de Léséleuc, V. Lienhard, T. Lahaye, and A. Browaeys, An Atom-by-Atom Assembler of Defect-Free Arbitrary 2D Atomic Arrays, Science 354, 1021 (2016).

[41] R. M. W. van Bijnen, Quantum Engineering with Ultracold Atoms, Ph.D. thesis, Technische Universiteit Eindhoven, 2011.

[42] S. Whitlock, A. W. Glaetzle, and P. Hannaford, Simulating Quantum Spin Models Using Rydberg-Excited Atomic Ensembles in Magnetic Microtrap Arrays, J. Phys. B 50, 074001 (2017).

[43] D. Barredo, H. Labuhn, S. Ravets, T. Lahaye, A. Browaeys, and C.S. Adams, Coherent Excitation Transfer in a Spin
Chain of Three Rydberg Atoms, Phys. Rev. Lett. 114, 113002 (2015).

[44] I. I. Beterov, I. I. Ryabtsev, D. B. Tretyakov, and V. M. Entin, Quasiclassical Calculations of BlackbodyRadiation-Induced Depopulation Rates and Effective Lifetimes of Rydberg $n s, n p$, and nd Alkali-Metal Atoms with $n \leq 80$, Phys. Rev. A 79, 052504 (2009).

[45] M. Marcuzzi, J. Minář, D. Barredo, S. de Léséleuc, H. Labuhn, T. Lahaye, A. Browaeys, E. Levi, and I. Lesanovsky, Facilitation Dynamics and Localization Phenomena in Rydberg Lattice Gases with Position Disorder, Phys. Rev. Lett. 118, 063606 (2017).

[46] P. W. Anderson, Absence of Diffusion in Certain Random Lattices, Phys. Rev. 109, 1492 (1958).

[47] P. Schauß, M. Cheneau, M. Endres, T. Fukuhara, S. Hild, A. Omran, T. Pohl, C. Gross, S. Kuhr, and I. Bloch, Observation of Spatially Ordered Structures in a Two-Dimensional Rydberg Gas, Nature (London) 491, 87 (2012).

[48] T. Caneva, T. Calarco, and S. Montangero, Chopped Random-Basis Quantum Optimization, Phys. Rev. A 84, 022326 (2011). 